\title{
Bioinformatics Analysis of the Interaction between Coat Protein and Nuclear Shuttle Protein in Babuvirus
}

\author{
Naitong Yu ${ }^{1,2^{*}}$, Jianhua Wang1,2, Naixin Yu ${ }^{1,2}$, Xiaobao Zheng1,2, Qin Zhou ${ }^{1,2}$, Zhixin Liu ${ }^{1,2^{*}}$ \\ ${ }^{1}$ Key Laboratory of Biology and Genetic Resources of Tropical Crops, Ministry of Agriculture and Rural Affairs, Institute of \\ Tropical Bioscience and Biotechnology, Chinese Academy of Tropical Agricultural Sciences, Haikou, China \\ ${ }^{2}$ Hainan Provincial Key Laboratory of Microbiology, Haikou, China \\ Email: *yunaitong@163.com, *liuzhixin@itbb.org.cn
}

How to cite this paper: $Y u$, N.T., Wang, J.H., Yu, N.X., Zheng, X.B., Zhou, Q. and Liu, Z.X. (2019) Bioinformatics Analysis of the Interaction between Coat Protein and Nuclear Shuttle Protein in Babuvirus. American Journal of Plant Sciences, 10, 622-630

https://doi.org/10.4236/ajps.2019.104045

Received: March 19, 2019

Accepted: April 23, 2019

Published: April 26, 2019

Copyright $\odot 2019$ by author(s) and Scientific Research Publishing Inc. This work is licensed under the Creative Commons Attribution International License (CC BY 4.0).

http://creativecommons.org/licenses/by/4.0/

\begin{abstract}
Protein and protein interactions play important roles in many biological processes and are responsible for carrying out the function of biological regulatory network in living organisms. Previous study indicated that Banana bunchy top virus (BBTV) coat protein (CP) interacted with BBTV nuclear shuttle protein (NSP). However, the protein and protein interaction and the binding affinity of CP and NSP in Babuvirus are remaining unclear. In this study, the CPs and NSPs proteins of BBTV, Abaca bunchy top virus (ABTV) and Cardamom bushy dwarf virus (CBDV) were used for bioinformatic analysis. The binding free energy and the dissociation constant of the possible interaction proteins were tested in PPA-Pred2, and the results confirmed CP interaction with NSP in Babuvirus. The study will help us to understand the interaction between viral protein and viral protein, and the pathogenesis mechanism of Babuvirus in host plants.
\end{abstract}

\section{Keywords}

Babuvirus, Coatprotein, Nuclear Shuttle Protein, Interaction, Bioinformatics Analysis

\section{Introduction}

Banana bunchy top virus (BBTV) belongs to the genus Babuvirus within the family Nanoviridae [1] [2]. The BBTV-induced banana bunchy top disease (BBTD) can cause significant economic losses in banana industry [3] [4]. Its genome contains at least six circular single-stranded DNA molecules with 1.0 - 1.1 $\mathrm{kb}$ in size, which names DNA-R, -U3, $-\mathrm{S},-\mathrm{M},-\mathrm{C},-\mathrm{N}$, respectively [5] [6]. Some 
isolates may also carry 1 - 3 distant satellite molecules which have similar function as DNA-R component [7] [8]. Each component is encapsulated into an icosahedral particle of $17-20 \mathrm{~nm}$ that has no envelope [9]. BBTV is transmitted by the banana aphid, Pentalonia nigronervosa [10] [11].

DNA-S encodes a coat protein $(\mathrm{CP})$ which encapsidates each DNA component inside [12]. Meanwhile, $\mathrm{CP}$ protein is a viral silencing suppressor that promotes the virus infection in host plants [13]. DNA-N encodes a nuclear shuttle protein (NSP) which can translocate the viral protein or protein and viral DNA complexes out of the cell nucleus [14]. Furthermore, the NSP protein contains an "FNGSF" motif that inhibits plant stress granules (SG) [15]. In vitro experiments showed that both CP and NSP proteins locate in the cytoplasm and nucleus of banana protoplast cell, and movement protein (MP) is able to re-locate NSP protein or NSP-DNA complex around the cell periphery, but not re-locate of the CP protein [14]. In vivo experiments showed the CP locates in the cell nucleus of Nicotiana benthamiana, while the NSP distributes in the cell nucleus and cytoplasm. Co-localization indicated that BBTV NSP interacts and re-distributes BBTV CP in tobacco cells [16].

According to the classification report of International Committee on Taxonomy of Viruses (ICTV, 2017), the family of Nanoviridae can be divided into the genus of Babuvirus and the genus of Nanovirus [1]. Currently, Nanovirus includes Black medic leaf roll virus (BMLV), Faba bean necrotic stunt virus (FBNSV), Faba bean necrotic yellows virus (FBNYV), Faba bean yellow leaf virus (FBYLV), Milk vetch dwarf virus (MVDV), Pea necrotic yellow dwarf virus (PNYDV), Pea yellow stunt virus (PYSV), and Subterranean clover stunt virus (SCSV), while Babuvirus includes BBTV, Abaca bunchy top virus (ABTV), and Cardamom bushy dwarf virus (CBDV).

Protein and protein interactions play important roles in many biological processes and are responsible for carrying out the function of biological regulatory network in living organisms. Previous study indicated that BBTV CP interacted with BBTV NSP [16]. However, the protein and protein interaction and the binding affinity of CP and NSP in Babuvirus are remaining unclear. In this study, the CPs and NSPs proteins of BBTV, ABTV, and CBDV were used for bioinformatic analysis. The study will help us to understand the interaction between viral protein and viral protein, and the pathogenesis mechanism of Babuvirus in host plants.

\section{Materials and Methods}

\subsection{Materials}

BBTV infected B2 sample (Haikou, China) was stored in Laboratory of Molecular Virology, Institute of Tropical Bioscience and Biotechnology (ITBB), Chinese Academy of Tropical Agricultural Sciences (CATAS). The complete nucleotide sequences of DNA-S (GenBank accession No. MG545612) and DNA-N (GenBank accession No. MG545615) were downloaded from the GenBank database 
in National Center for Biotechnology Information (NCBI,

https://www.ncbi.nlm.nih.gov).

\subsection{Nucleotide and Deduced Amino Acid Sequences of CP in Nanoviridae}

The nucleotide sequences of BBTV $C P$ (HQ616076.1), ABTV $C P$ (FJ787435.1), CBDV $C P$ (JX867540.1), FBNSV $C P$ (GQ274036.1), PNYDV $C P$ (KC979044.1), MVDV $C P$ (LN890467.1), BMLV $C P$ (NC_023304.1), FBNYV $C P$ (GQ274028.1), FBYLV $C P$ (HE654124.1) and PYSV $C P$ (NC_023308.1) were downloaded from the GenBank database in NCBI. The amino acid sequences of BBTV CP (AEF97830.1), ABTV CP (ACN79534.1), CBDV CP (AGG38924.1), FBNSV CP (ACX50514.1), PNYDV CP (AHC72272.1), MVDV CP (CUR70740.1), BMLV CP (YP_008997802.1), FBNYV CP (ACX50506.1), FBYLV CP (CCF74114.1) and PYSV CP (YP_008997806.1) were downloaded from the GenBank database in NCBI as well. Sequence alignment was performed by using BioEdit (version 7.0.9.0). The sequence identities of the nucleotide and amino acid were analyzed by the Sequence Identity Matrix program in BioEdit.

\subsection{Nucleotide and Deduced Amino Acid Sequences of NSP in Nanoviridae}

The nucleotide sequences of BBTV NSP (FJ463047.1), ABTV NSP(NC_010314.1), CBDV NSP (JX867546.1), FBNSV NSP(GQ274038.1), PNYDV NSP(KC979047.1), MVDV NSP (NC_003643.1), BMLV NSP (NC_023301.1), FBNYV NSP (NC_003566.1), FBYLV NSP (HE654127.1) and PYSV NSP (NC_023310.1) were downloaded from the GenBank database in NCBI. The amino acid sequences of BBTV NSP (ACK43793.1), ABTV NSP (YP_001661656.1), CBDV NSP (AGG38930.1), FBNSV NSP (ACX50516.1), PNYDV NSP (AHC72275.1), MVDV NSP (NP_619764.1), BMLV NSP (YP_008997799.1), FBNYV NSP (NP_619573.1), FBYLV NSP (CCF74117.1) and PYSV NSP (YP_008997808.1) were downloaded from the GenBank database in NCBI as well. Sequence alignment was performed by using BioEdit (version 7.0.9.0). The sequence identities of the nucleotide and amino acid were also analyzed by the Sequence Identity Matrix program in BioEdit.

\subsection{The Prediction of Interaction between CP and NSP Proteins in Babuvirus}

Previous study indicated that BBTV CP interacted with BBTV NSP [16]. In order to determine the binding affinity of the two proteins, the amino acid sequences of BBTV CP (AEF97830.1) and BBTV NSP (ACK43793.1) were input into PPA-Pred2 (http://www.iitm.ac.in/bioinfo/PPA_Pred/index.html) for binding affinity analysis using jack-knife test [17]. The relationship between ABTV CP (ACN79534.1) and ABTV NSP (YP_001661656.1) and the relationship between CBDV CP (AGG38924.1) and CBDV NSP (AGG38930.1) were also conducted. The predicted value of Delta G (binding free energy) and the predicted value of 
Kd (dissociation constant) were used to evaluate the interaction.

\section{Results}

\subsection{Sequences Identity of Nucleotide and Amino Acids of CP in Nanoviridae}

The complete ORF of BBTV $C P$ is $513 \mathrm{bp}$, and its encoded protein is 170 aa with predicted molecular mass of about $19.35 \mathrm{kDa}$ in size. Nucleotide sequence analysis showed that BBTV $C P$ gene shares $34.5 \%$ - 97.8\% identity with other plant viruses in Nanoviridae family. Among them, it shared $97.8 \%$ and $71.0 \%$ homology with ABTV and CBDV, respectively, while it was only $34.5 \%-40.0 \%$ identity with Nanovirus. Amino acid sequences identity showed that BBTV CP protein is as high as $97.6 \%$ to ABTV, and $75.2 \%$ homologous to CBDV. However, the homology between the BBTV CP and Nanovirus CPs was less than 21.8\% (Table 1 and Figure 1).

\subsection{Sequences Identity of Nucleotide and Amino Acids of NSP in Nanoviridae}

The complete ORF of BBTV NSP is $465 \mathrm{bp}$, and its encoded protein is 154 aa with predicted molecular mass of about $17.55 \mathrm{kDa}$ in size. The sequence identity analysis of BBTV NSP gene showed that it both shares $\sim 70 \%$ homology with ABTV and CBDV, and it was about 50\% homology with Nanovirus. Amino acid sequence identity also found that it shares about $70 \%$ homology with ABTV and CBDV, while about $45 \%$ homology with Nanovirus, a little lower than nucleotide sequence identity (Table 2 and Figure 2). Furthermore, the "FNGSF" motif was conserved in Nanoviridae (Figure 2), which plays the role of inhibiting plant stress granules (SG) formation.

Table 1. Nucleotide and amino acid sequence identity analyses of BBTV $C P$ gene with other $C P$ genes of nanovirids.

\begin{tabular}{ccccccccccc}
\hline Gene/Protein & BBTV CP & ABTV CP & CBDV CP & FBNSV CP & PNYDV CP & MVDV CP & BMLV CP & FBNYV CP & FBYLV CP & PYSV CP \\
\hline BBTV CP & - & 0.978 & 0.71 & 0.4 & 0.379 & 0.388 & 0.378 & 0.39 & 0.394 \\
ABTV CP & $\mathbf{0 . 9 7 6}$ & - & 0.717 & 0.402 & 0.381 & 0.387 & 0.384 & 0.383 & 0.385 & 0.345 \\
CBDV CP & $\mathbf{0 . 7 5 2}$ & $\mathbf{0 . 7 6 4}$ & - & 0.392 & 0.387 & 0.37 & 0.382 & 0.387 & 0.396 & 0.354 \\
FBNSV CP & $\mathbf{0 . 2 1 8}$ & $\mathbf{0 . 2 1 8}$ & $\mathbf{0 . 2 2 4}$ & - & 0.607 & 0.801 & 0.652 & 0.782 & 0.824 & 0.644 \\
PNYDV CP & $\mathbf{0 . 2 1 8}$ & $\mathbf{0 . 2 1 8}$ & $\mathbf{0 . 2 3 5}$ & $\mathbf{0 . 5 5 8}$ & - & 0.59 & 0.528 & 0.59 & 0.605 & 0.58 \\
MVDV CP & $\mathbf{0 . 2 0 1}$ & $\mathbf{0 . 2 0 1}$ & $\mathbf{0 . 1 8 9}$ & $\mathbf{0 . 8 6}$ & $\mathbf{0 . 5 2 9}$ & - & 0.644 & 0.793 & 0.815 & 0.653 \\
BMLV CP & $\mathbf{0 . 2}$ & $\mathbf{0 . 2}$ & $\mathbf{0 . 2 0 5}$ & $\mathbf{0 . 5 6 8}$ & $\mathbf{0 . 4 3 1}$ & $\mathbf{0 . 5 8 6}$ & - & 0.642 & 0.667 & 0.608 \\
FBNYV CP & $\mathbf{0 . 1 9 5}$ & $\mathbf{0 . 1 9 5}$ & $\mathbf{0 . 1 9 5}$ & $\mathbf{0 . 8 4 8}$ & $\mathbf{0 . 5 2 9}$ & $\mathbf{0 . 8 1 9}$ & $\mathbf{0 . 5 5 1}$ & - & 0.824 & 0.651 \\
FBYLV CP & $\mathbf{0 . 1 8 3}$ & $\mathbf{0 . 1 8 3}$ & $\mathbf{0 . 1 8 3}$ & $\mathbf{0 . 8 8 3}$ & $\mathbf{0 . 5 2 3}$ & $\mathbf{0 . 8 3 7}$ & $\mathbf{0 . 5 8 6}$ & $\mathbf{0 . 8 8 9}$ & - \\
PYSV CP & $\mathbf{0 . 1 7 8}$ & $\mathbf{0 . 1 7 8}$ & $\mathbf{0 . 1 7 2}$ & $\mathbf{0 . 5 8 7}$ & $\mathbf{0 . 4 9 1}$ & $\mathbf{0 . 5 8 1}$ & $\mathbf{0 . 5 5 7}$ & $\mathbf{0 . 5 8 1}$ & $\mathbf{0 . 5 8 7}$ & - \\
\hline
\end{tabular}

Note: The bold numbers represent the amino acid sequence identity matrix; un-bold numbers represent the nucleotide sequence identity matrix. 
N. T. Yu et al.

Table 2. Nucleotide and amino acid sequence identity analyses of BBTV NSP gene with other NSP genes of nanovirids.

\begin{tabular}{lllllllllll}
\hline Gene/Protein & BBTV NSP & ABTV NSP & CBDV NSP & $\begin{array}{c}\text { FBNSV } \\
\text { NSP }\end{array}$ & $\begin{array}{c}\text { PNYDV } \\
\text { NSP }\end{array}$ & $\begin{array}{c}\text { MVDV } \\
\text { NSP }\end{array}$ & BMLV NSP & $\begin{array}{c}\text { FBNYV } \\
\text { NSP }\end{array}$ & $\begin{array}{c}\text { FBYLV } \\
\text { NSP }\end{array}$ & PYSV NSP \\
\hline BBTV NSP & - & 0.709 & 0.72 & 0.518 & 0.486 & 0.49 & 0.515 & 0.479 & 0.52 & 0.513 \\
ABTV NSP & $\mathbf{0 . 7 0 1}$ & - & 0.72 & 0.511 & 0.481 & 0.494 & 0.49 & 0.471 & 0.513 & 0.524 \\
CBDV NSP & $\mathbf{0 . 7 0 3}$ & $\mathbf{0 . 6 7 7}$ & - & 0.484 & 0.479 & 0.473 & 0.503 & 0.462 & 0.479 & 0.501 \\
FBNSV NSP & $\mathbf{0 . 4 4 2}$ & $\mathbf{0 . 4 6 1}$ & $\mathbf{0 . 4 5 2}$ & - & 0.714 & 0.829 & 0.766 & 0.833 & 0.88 & 0.79 \\
PNYDV NSP & $\mathbf{0 . 4 2 3}$ & $\mathbf{0 . 4 4 2}$ & $\mathbf{0 . 4 1 4}$ & $\mathbf{0 . 7 5 1}$ & - & 0.72 & 0.705 & 0.725 & 0.72 & 0.725 \\
MVDV NSP & $\mathbf{0 . 4 6 1}$ & $\mathbf{0 . 4 8}$ & $\mathbf{0 . 4 5 2}$ & $\mathbf{0 . 8 8 8}$ & $\mathbf{0 . 7 5 1}$ & - & 0.759 & 0.839 & 0.837 & 0.768 \\
BMLV NSP & $\mathbf{0 . 4 4 8}$ & $\mathbf{0 . 4 4 2}$ & $\mathbf{0 . 4 2 6}$ & $\mathbf{0 . 8 0 3}$ & $\mathbf{0 . 7 1 8}$ & $\mathbf{0 . 8 0 3}$ & - & 0.761 & 0.77 & 0.751 \\
FBNYV NSP & $\mathbf{0 . 4 4 8}$ & $\mathbf{0 . 4 5 5}$ & $\mathbf{0 . 4 3 9}$ & $\mathbf{0 . 8 8 8}$ & $\mathbf{0 . 7 4 5}$ & $\mathbf{0 . 9 0 8}$ & $\mathbf{0 . 7 9}$ & - & 0.839 & 0.766 \\
FBYLV NSP & $\mathbf{0 . 4 5 5}$ & $\mathbf{0 . 4 6 1}$ & $\mathbf{0 . 4 5 2}$ & $\mathbf{0 . 9 0 8}$ & $\mathbf{0 . 7 3 8}$ & $\mathbf{0 . 9 0 8}$ & $\mathbf{0 . 7 9}$ & $\mathbf{0 . 8 9 5}$ & - & 0.809 \\
PYSV NSP & $\mathbf{0 . 4 6 1}$ & $\mathbf{0 . 4 7 4}$ & $\mathbf{0 . 4 3 3}$ & $\mathbf{0 . 8 1 6}$ & $\mathbf{0 . 7 3 8}$ & $\mathbf{0 . 8 2 3}$ & $\mathbf{0 . 7 9}$ & $\mathbf{0 . 8 2 3}$ & $\mathbf{0 . 8 4 3}$ & - \\
\hline
\end{tabular}

Note: The bold numbers represent the amino acid sequence identity matrix; un-bold numbers represent the nucleotide sequence identity matrix.
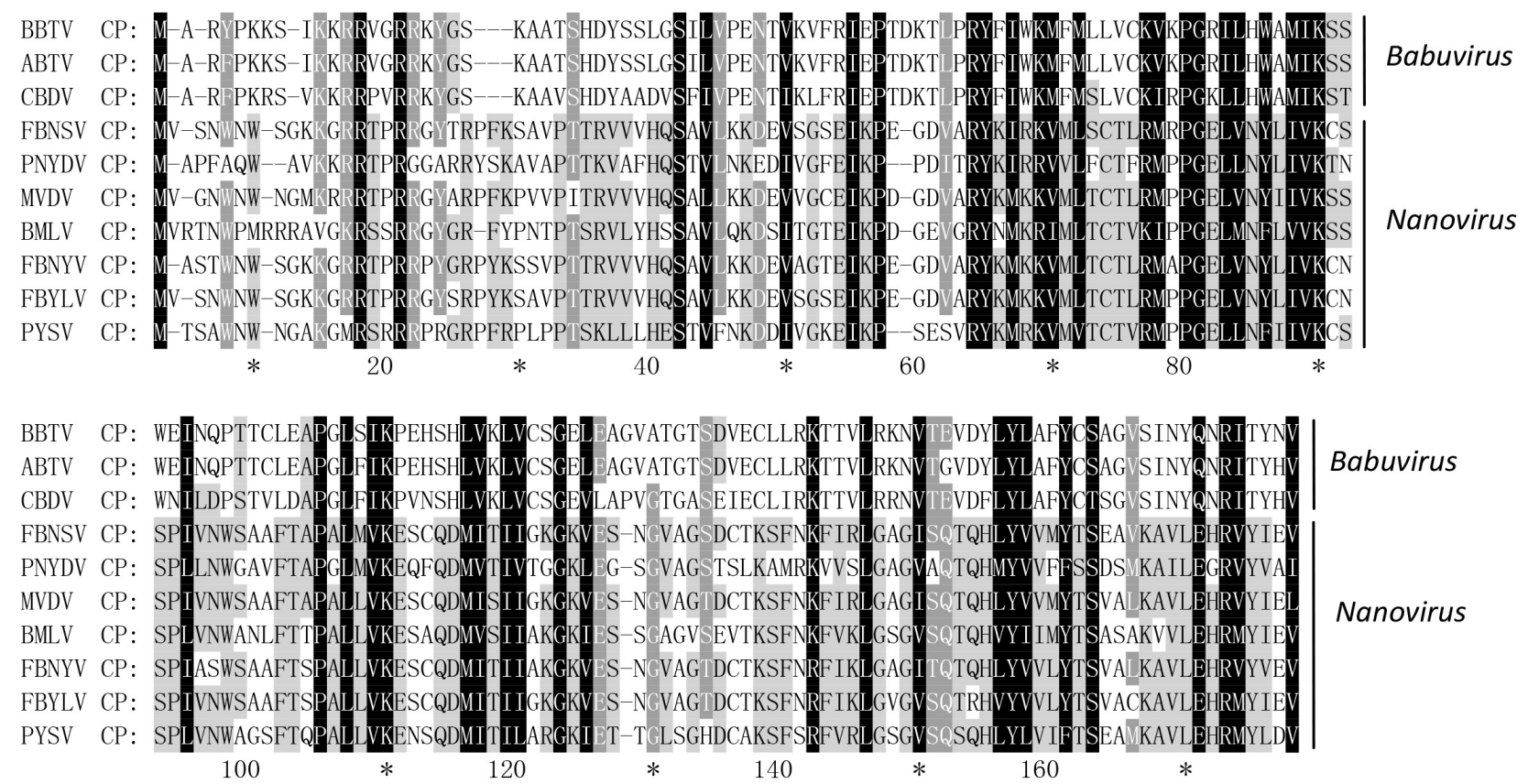

Figure 1. Multiple amino acid sequence alignment of BBTV CP with other CP of nanovirids. The black shaded regions indicate conserved residues, while the grey shaded regions are partially conserved residues with greater than $80 \%$ conservation. The numerical labeling on the residues indicated the total number of the amino acids at the corresponding position. GenBank accession numbers of the sequences used for the above analysis are as follows: BBTV CP, AEF97830.1; ABTV CP, ACN79534.1; CBDV CP, AGG38924.1; FBNSV CP, ACX50514.1; PNYDV CP, AHC72272.1; MVDV CP, CUR70740.1; BMLV CP, YP_008997802.1; FBNYV CP, ACX50506.1; FBYLV CP, CCF74114.1; PYSV CP, YP_008997806.1.

\subsection{The Prediction of Interaction between CP and NSP Proteins in Babuvirus}

PPA-Pred2 analysis indicated that BBTV CP had binding affinity with BBTV NSP. In detail, the predicted value of Delta G (binding free energy) was -11.69 

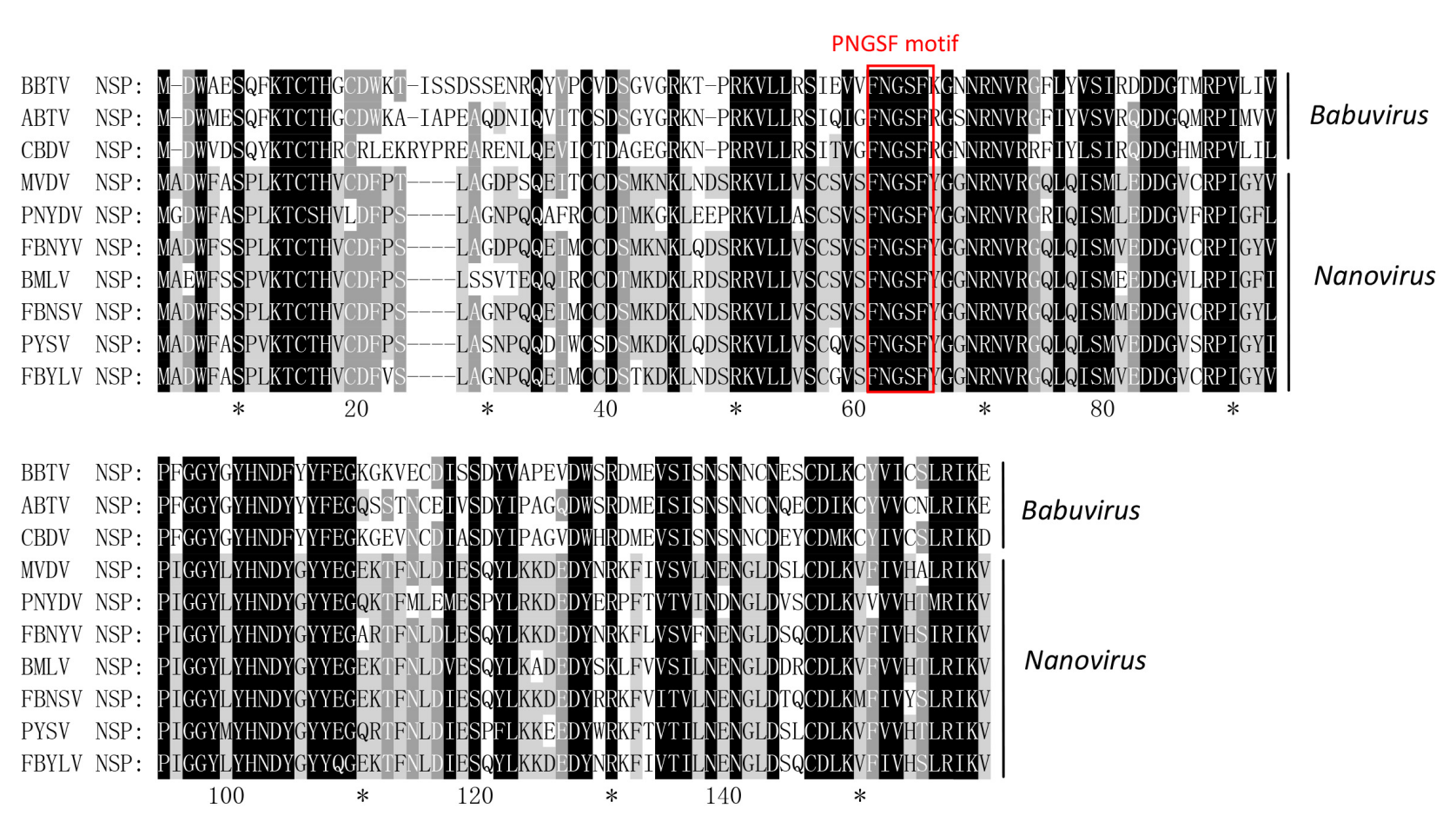

Figure 2. Multiple amino acid sequence alignment of BBTV NSP with other NSP of nanovirids. The black shaded regions indicate conserved residues, while the grey shaded regions are partially conserved residues with greater than $80 \%$ conservation. The numerical labeling on the residues indicated the total number of the amino acids at the corresponding position. GenBank accession numbers of the sequences used for the above analysis are as follows: BBTV NSP, ACK43793.1; ABTV NSP, YP_001661656.1; CBDV NSP, AGG38930.1; FBNSV NSP, ACX50516.1; PNYDV NSP, AHC72275.1; MVDV NSP, NP_619764.1; BMLV NSP, YP_008997799.1; FBNYV NSP, NP_619573.1; FBYLV NSP, CCF74117.1; PYSV NSP, YP_008997808.1.

$\mathrm{kcal} / \mathrm{mol}$, and the predicted value of $\mathrm{Kd}$ (dissociation constant) was $2.65 \mathrm{e}^{-09} \mathrm{M}$. Meanwhile, the bioinformatic analysis confirmed the interaction between ABTV $\mathrm{CP}$ and ABTV NSP. The predicted value of Delta G (binding free energy) was $-11.99 \mathrm{kcal} / \mathrm{mol}$ and the predicted value of $\mathrm{Kd}$ (dissociation constant) was $1.61 \mathrm{e}^{-09} \mathrm{M}$. Lastly, the bioinformatic analysis confirmed the interaction between CBDV CP and CBDV NSP as well. The predicted value of Delta G (binding free energy) was $-8.92 \mathrm{kcal} / \mathrm{mol}$ and the predicted value of $\mathrm{Kd}$ (dissociation constant) was $2.88 \mathrm{e}^{-07} \mathrm{M}$. Therefore, the result confirmed CP interaction with NSP in Babuvirus.

\section{Discussion}

Protein and protein interactions are crucial bridges for many biological processes involved in cellular signaling, immunity, cellular transport, etc. [18] [19] [20]. Experimentally, protein and protein interactions have been mainly studied with the yeast two-hybrid system, GST pull-down, co-immunoprecipitation (Co-IP), co-localization and so on. In addition, the fluorescence resonance energy transfer (FRET), isothermal titration calorimetry (ITC), surface plasmon resonance (SPR) and PPA-Pred2 provide the affinity of interacting proteins in terms of binding free energy change and dissociation constant [21] [22] [23], thereby adding a new dimension analysis of the task of protein and protein inte- 
raction network. Previous study indicated that BBTV CP interacted with BBTV NSP. In this work, the possible interaction of CP protein and NSP protein of BBTV, ABTV and CBDV was further predicted based on their amino acid sequences. The results confirmed CP interaction with NSP in Babuvirus by PPA-Pred2 analysis.

The interaction between CP and NSP in Babuvirus was well predicted by PPA-Pred2 analysis based on the amino acid sequences, not by experiments. However, this method has a few limitations. For example, the protein whose structure is unclear or the protein which needs further processing modification could not be widely used in this method. In addition, the method cannot be used for prediction the binding affinity of more than two proteins.

In this study, the NSP is a vial nuclear shuttle protein, which would help viral proteins or viral nucleic acid transport outside of the nucleus by interaction with MP [14]. Furthermore, the "FNGSF" motif that inhibits plant stress granules (SG) was conserved in all nanovirids NSPs [15]. It is speculated that Babuvirus NSP proteins have similar functions. The product of the Babuvirus $C P$ gene is an important structural protein that constitutes the virion. Meanwhile, $\mathrm{CP}$ protein is a viral silencing suppressor that promotes the virus infection in the host plant. BBTV NSP affects the BBTV CP distribution by co-localization analysis, suggesting that BBTV NSP interacts and re-locates the BBTV CP in tobacco cells. During the virus infection, Babuvirus NSP would re-locate the Babuvirus CP, which plays the key role of interaction between virus and host, such as inhibition the host antiviral activity by CP. Therefore, the interaction of CP and NSP viral proteins would play a very important role in the Babuvirus infection or systemic infection, and further studies should be conducted.

\section{Funding}

This work was supported by the Hainan Provincial Natural Science Foundation (20153130), the National Natural Science Foundation of China (31401709), the Young Elite Scientists Sponsorship Program by CSTC (CSTC-QN201704) and Central Public-interest Scientific Institution Basal Research Fund for Chinese Academy of Tropical Agricultural Sciences (No.19CXTD-33).

\section{Conflicts of Interest}

The authors declare no conflicts of interest regarding the publication of this paper.

\section{References}

[1] Lefkowitz, E.J., Dempsey, D.M., Hendrickson, R.C., Orton, R.J., Siddell, S.G. and Smith, D.B. (2018) Virus Taxonomy: The Database of the International Committee on Taxonomy of Viruses (ICTV). Nucleic Acids Research, 46, D708-D717. https://doi.org/10.1093/nar/gkx932

[2] Qazi, J. (2016) Banana Bunchy Top Virus and the Bunchy Top Disease. Journal of General Plant Pathology, 82, 2-11. https://doi.org/10.1007/s10327-015-0642-7 
[3] Kumar, P.L., Selvarajan, R., Iskra-Caruana, M.L., Chabannes, M. and Hanna, R. (2015) Chapter Seven Biology, Etiology, and Control of Virus Diseases of Banana and Plantain. Advances in Virus Research, 91, 229. https://doi.org/10.1016/bs.aivir.2014.10.006

[4] Yu, N.T. and Liu, Z.X. (2011) New Research Advance of Banana Bunchy Top Virus. Microbiology China, 38, 396-404.

[5] Burns, T.M., Harding, R.M. and Dale, J.L. (1995) The Genome Organization of Banana Bunchy Top Virus: Analysis of Six ssDNA Components. Journal of General Virology, 76, 1471-1482. https://doi.org/10.1099/0022-1317-76-6-1471

[6] Yu, N.T., Zhang, Y.L., Feng, T.C., Wang, J.H., Kulye, M., Yang, W.J., Lin, Z.S., Xiong, Z. and Liu, Z.X. (2012) Cloning and Sequence Analysis of Two Banana Bunchy Top Virus, Genomes in Hainan. Virus Genes, 44, 488-494.

https://doi.org/10.1007/s11262-012-0718-9

[7] Stainton, D., Martin, D.P., Collings, D.A. and Varsani, A. (2016) Comparative Analysis of Common Regions Found in Babuviruses and Alphasatellite Molecules. Archives of Virology, 162, 1-7.

[8] Yu, N.T., Feng, T.C., Zhang, Y.L., Wang, J.H. and Liu, Z.X. (2011) Bioinformatic Analysis of BBTV Satellite DNA in Hainan. Virologica Sinica, 26, 279-284. https://doi.org/10.1007/s12250-011-3196-7

[9] Gronenborn, B. (2004) Nanoviruses: Genome Organisation and Protein Function. Veterinary Microbiology, 98, 103-109. https://doi.org/10.1016/j.vetmic.2003.10.015

[10] Watanabe, S. and Bressan, A. (2013) Tropism, Compartmentalization and Retention of Banana Bunchy Top Virus (Nanoviridae) in the Aphid Vector Pentalonia Nigronervosa. Journal of General Virology, 94, 691-697. https://doi.org/10.1099/vir.0.047308-0

[11] Bressan, A. and Watanabe, S. (2011) Immunofluorescence Localisation of Banana Bunchy Top Virus (Family Nanoviridae) within the Aphid Vector, Pentalonia Nigronervosa, Suggests a Virus Tropism Distinct from Aphid-Transmitted Luteoviruses. Virus Research, 155, 520-525. https://doi.org/10.1016/j.virusres.2010.12.005

[12] Wanitchakorn, R., Harding, R.M. and Dale, J.L. (1997) Banana Bunchy Top Virus DNA-3 Encodes the Viral Coat Protein. Archives of Virology, 142, 1673-1680. https://doi.org/10.1007/s007050050188

[13] Niu, S., Wang, B., Guo, X., Yu, J., Wang, X., Xu, K., Zhai, Y., Wang, J. and Liu, Z. (2009) Identification of Two RNA Silencing Suppressors from Banana Bunchy Top Virus. Archives of Virology, 154, 1775-1783. https://doi.org/10.1007/s00705-009-0515-5

[14] Wanitchakorn, R., Hafner, G.J., Harding, R.M. and Dale, J.L. (2000) Functional Analysis of Proteins Encoded by Banana Bunchy Top Virus DNA-4 to -6. Journal of General Virology, 81, 299-306. https://doi.org/10.1099/0022-1317-81-1-299

[15] Krapp, S., Greiner, E., Amin, B., Sonnewald, U. and Krenz, B. (2017) The Stress Granule Component g3bp Is a Novel Interaction Partner for the Nuclear Shuttle Proteins of the Nanovirus Pea Necrotic Yellow Dwarf Virus and Geminivirus Abutilon Mosaic Virus. Virus Research, 227, 6-14. https://doi.org/10.1016/j.virusres.2016.09.021

[16] Ji, X.L., Yu, N.T., Qu, L., Li, B.B. and Liu, Z.X. (2019) Banana Bunchy Top Virus (BBTV) Nuclear Shuttle Protein Interacts and Re-Distributes BBTV Coat Protein in Nicotiana benthamiana. 3 Biotech, 9, 121. https://doi.org/10.1007/s13205-019-1656-1

[17] Yugandhar, K. and Gromiha, M.M. (2014) Protein-Protein Binding Affinity Predic- 
tion from Amino Acid Sequence. Bioinformatics, 30, 3583-3589.

https://doi.org/10.1093/bioinformatics/btu580

[18] Levskaya, A., Weiner, O.D., Lim, W.A. and Voigt, C.A. (2009) Spatiotemporal Control of Cell Signaling Using a Light-Switchable Protein Interaction. Nature, 461, 997-1001. https://doi.org/10.1038/nature08446

[19] Mei, S., Flemington, E.K. and Zhang, K. (2018) Transferring Knowledge of Bacterial Protein Interaction Networks to Predict Pathogen Targeted Human Genes and Immune Signaling Pathways: A Case Study on M. tuberculosis. BMC Genomics, 19, 505. https://doi.org/10.1186/s12864-018-4873-9

[20] Cheng, H., Burroughs-Garcia, J., Birkness, J.E., Trinidad, J.C. and Deans, M.R. (2016) Disparate Regulatory Mechanisms Control Fat3 and P75NTR Protein Transport through a Conserved Kif5-Interaction Domain. PLOS ONE, 11, e0165519. https://doi.org/10.1371/journal.pone.0165519

[21] Qian, Y., Zhou, L., Wu, Y.X., Zhang, K., Cao, Y., Zhou, Y., Wu, D., Hu, F. and Gan, N. (2018) A Two Dimensional Metal-Organic Framework Nanosheets-Based Fluorescence Resonance Energy Transfer Aptasensor with Circular Strand-Replacement DNA Polymerization Target-Triggered Amplification Strategy for Homogenous Detection of Antibiotics. Analytica Chimica Acta, 1020, 1-8.

https://doi.org/10.1016/j.aca.2018.02.058

[22] Lin, K. and Wu, G. (2019) Isothermal Titration Calorimetry Assays to Measure Binding Affinities in Vitro. Methods in Molecular Biology, 1893, 257-272.

https://doi.org/10.1007/978-1-4939-8910-2_19

[23] Chiu, N.F. and Lin, T.L. (2018) Affinity Capture Surface Carboxyl-Functionalized MoS 2 Sheets to Enhance the Sensitivity of Surface Plasmon Resonance Immunosensors. Talanta, 185, 174-181. https://doi.org/10.1016/j.talanta.2018.03.073 